\title{
Centrosome aberration accompanied with p53 mutation can induce genetic instability in hepatocellular carcinoma
}

\author{
Tomoki Nakajima ${ }^{1}$, Michihisa Moriguchi ${ }^{1}$, Yasuhide Mitsumoto ${ }^{1}$, Satoru Sekoguchi ${ }^{1}$, \\ Taichirou Nishikawa ${ }^{1}$, Hidetaka Takashima ${ }^{1}$, Tadashi Watanabe ${ }^{1}$, Tatsuo Katagishi ${ }^{1}$, \\ Hiroyuki Kimura $^{1}$, Takeshi Okanoue ${ }^{1}$ and Keizo Kagawa ${ }^{2}$

\begin{abstract}
${ }^{1}$ Molecular Gastroenterology and Hepatology, Kyoto Prefectural University of Medicine Graduate School of Medical Science, Kyoto, Japan and ${ }^{2}$ Department of Internal Medicine, Fukuchiyama City Hospital, Fukuchiyama, Japan
\end{abstract}

\begin{abstract}
Centrosome duplication is controlled in a cell cycle-specific manner and occurs once every cell cycle, thereby ensuring the balanced segregation of chromosomes during the mitotic phase. Numerical or structural abnormalities can arise in the centrosomes of malignant cells. Under defective cell cycle checkpoint systems, cancer cells with abnormal centrosomes can survive and re-enter the cell cycle, promoting unbalanced chromosome segregation and genetic instability. We investigated the centrosome aberrations in 33 patients diagnosed with hepatocellular carcinoma (HCC), using fluorescent pericentrin immunostaining. We also studied the p53 mutation, proliferative activity, and DNA ploidy in these cases. In normal hepatocytes, one centrosome was identified per cell as a round dot, usually in the vicinity of the nuclear membrane. However, in cancer cells from HCC tissue, several patterns of centrosome abnormalities occurred, including supernumerary centrosomes and centrosomes with an abnormal shape and size. Although the frequency of abnormal centrosomes in each tissue was relatively low compared with previous reports in other cancers, nevertheless, centrosome aberration was found in 30 out of $33 \mathrm{HCC}$ tissues. The percentage of tumor cells with abnormal centrosomes was significantly higher in the nondiploid tumors $(15.8 \pm 15.9 \%)$ than in the diploid tumors $(5.4 \pm 5.1 \% 0)(P<0.05)$, and tended to be higher in the tumors with p53 mutation $(11.6 \pm 13.1 \%$ ) than in those with wild-type p53 $(5.6 \pm 6.8 \%$ ) . Furthermore, $82 \%$ of nondiploid tumors exhibited p53 mutation, whereas only $41 \%$ of diploid tumors showed p53 mutation. The percentage of tumor cells with centrosome abnormalities were not related to tumor stage, size or proliferative activity. Therefore, our results indicate that hepatic cancer cells, under centrosome aberration and a defective checkpoint system possibly caused by p53 mutation, have the potential for genetic instability and aggressive behavior. This potential effect occurs irrespective of the tumor size or stage.

Modern Pathology (2004) 17, 722-727, advance online publication, 26 March 2004; doi:10.1038/modpathol.3800115
\end{abstract}

Keywords: centrosome; p53; genetic instability; hepatocellular carcinoma

The centrosome represents the microtubule-organizing center of the cell. It consists of a pair of centrioles, oriented at right angles to each other, and the surrounding pericentriolar material. In the $\mathrm{G}_{0}$ phase of the cell cycle in animals, there is one centrosome in each cell. Centrosome duplication occurs only once during each cell cycle and is a

Correspondence: T Nakajima, Molecular Gastroenterology and Hepatology, Kyoto Prefectural University of Medicine Graduate School of Medical Science, Kawaramachi-Hirokoji, Kamigyo-ku, Kyoto 602-8566, Japan.

E-mail: tomnaka@silver.ocn.ne.jp

Received 30 July 2003; revised 31 December 2003; accepted 2 January 2004; published online 26 March 2004 highly regulated process. It is initiated at the $\mathrm{G}_{1} / \mathrm{S}$ boundary and is completed during the $\mathrm{G}_{2}$ phase. In normal cells, the duplicated centrosomes then migrate to opposite ends of the cell to serve as two mitotic spindle poles just before mitosis. This process ensures the balanced segregation of chromosomes during the mitotic phase., ${ }^{1,2}$

Abnormal centrosomes have been identified in malignant cells and can result in the assembly of aberrant mitotic spindles, leading to abnormal segregation of chromosomes during the mitotic phase. $^{2-5}$ This unbalanced segregation of chromosomes, concomitant with the activation of oncogenes and the loss of control of tumor suppressor genes, is involved in the initial stages of tumorigenesis. 
Unbalanced segregation of chromosomes is also involved in tumor progression and in the development of a metastatic potential. ${ }^{2,3}$

In particular, the loss or mutation of the p53 tumor suppressor gene, the most commonly mutated gene in human cancers, has important consequences on mitotic fidelity. One of the normal roles of p53 is to inhibit or block cell proliferation when damaged DNA is present. It has been shown that the loss of this crucial p53 tumor suppressor function induces abnormal amplification of centrosomes through an obligate failure of the $G_{1} / S$ and $G_{2} / M$ checkpoint systems that would normally prevent tetraploid cells from re-entering the cell cycle. ${ }^{6-9}$ Thus, as p53 loss can contribute to the propagation of damaged DNA in daughter cells through the inability to prevent the transmission of inaccurate genetic material, it is considered to be one of the major mechanisms for the emergence of aneuploidy in tumors with inactivated p53 protein. ${ }^{10,11}$ However, there are only a few reports on the relationship between p53 inactivation and centrosome aberration among clinical cases of cancers. ${ }^{12,13}$

In human hepatocellular carcinoma (HCC), functional loss of p53 is frequently observed in the advanced stage and in histologically poorly differentiated tissues. ${ }^{14}$ However, to date, there have been no reports of centrosome abnormalities in clinical cases of HCC. In this paper, we report for the first time centrosome aberrations in HCC and their relationship with p53 status and DNA ploidy. Secondly, we report the associations between the centrosome status and clinicopathological features of this disease.

\section{Materials and methods}

\section{Sample Preparation}

In all, 33 samples of HCC-containing tissue were obtained under general anesthesia from 21 male and 12 female patients who were previously diagnosed with this cancer. The patients were $61.9 \pm 7.8$ (mean \pm S.D.) years old and none had received preoperative chemotherapy. Eight patients were serologically positive for hepatitis B surface antigen (HBs-Ag) only, 23 were positive for antihepatitis C antibody (HCV-Ab) only, and two were positive for both HBs-Ag and HCV-Ab. According to the tumor node metastasis (TNM) staging system for HCC, four patients were classified as stage I, 19 as stage II, eight as stage III and two as stage IV. Controls for this study were histologically normal liver tissues from five patients who had undergone partial hepatectomy for metastatic liver cancer. Written, informed consent was obtained from all patients.

Each sample was fixed with $4 \%$ buffered paraformaldehyde at $4{ }^{\circ} \mathrm{C}$ overnight and embedded in paraffin. Three sections, each of $4 \mu \mathrm{m}$ thickness, and one $60 \mu \mathrm{m}$-thick section, were cut serially from each paraffin block. The $4 \mu \mathrm{m}$-thick sections were used for hematoxylin and eosin (HE) staining, and immunohistochemical staining for aberrant p53 protein, Ki-67 antigen to assess proliferative activity, and pericentrin to assess centrosome abnormality. ${ }^{4,15}$ The $60 \mu$ m-thick section was used for DNA ploidy analysis.

\section{Immunohistochemistry for Aberrant p53 Protein, Ki-67 Antigen and Pericentrin}

After deparaffinization, the $4 \mu$ m-thick sections were immersed in tap water and then preincubated with $2 \%$ fetal bovine serum (FBS) diluted in Tris-buffered saline (TBS) at room temperature for $10 \mathrm{~min}$. Immunohistochemical analysis of aberrant p53 protein was performed by the streptavidin-biotinperoxidase method, on one $4 \mu \mathrm{m}$ section using antip53 monoclonal antibody (DO-7; DAKO, Kyoto, Japan) as the primary antibody after autoclaving in $10 \mathrm{mM}$ citrate buffer $\left(\mathrm{pH} \mathrm{6.0)}\right.$ for $10 \mathrm{~min}$ at $120^{\circ} \mathrm{C}$. The slide was lightly counterstained with hematoxylin. We regarded p53 to be overexpressed when the positive cancer cell rate was greater than $10 \%$ after blindly scoring 1000-2000 tumor cells. Ki-67 antigen was detected also by the streptavidin-biotinperoxidase method using the second $4 \mu \mathrm{m}$ section and anti-Ki67 monoclonal antibody (MIB-1; Immunotech, Marseille, France) as the primary antibody. Proliferative activity was then assessed by the Ki-67positive index (\%) after blindly scoring 1000-2000 tumor cells. ${ }^{16}$

An antipericentrin polyclonal rabbit antibody (Covance, Princeton, NJ, USA) was applied to the third $4 \mu \mathrm{m}$ section overnight at a dilution of $1: 150$, after autoclaving in $10 \mathrm{mM}$ citrate buffer with $0.1 \%$ Tween 20 (pH 6.0) for $10 \mathrm{~min}$ at $120^{\circ} \mathrm{C}$. Pericentrin was detected by using fluorescein isothiocyanate (FITC)-conjugated anti-rabbit immunoglobulin (DAKO, Kyoto, Japan) as a secondary antibody, and examined with a fluorescence microscope after counterstaining with $4^{\prime}, 6$-diamidino-2-phenylindole (DAPI, Molecular Probes, Eugene, OR, USA). The original fluorescence images were preserved as digital images and analyzed, using IP Lab software (version 3.5, Scanalytics, Fairfax, VA, USA). To determine centrosome abnormalities in each section, at least 1000 cells were examined with respect to the number and diameter of pericentrin-staining dots. As previously reported ${ }^{17}$ centrosomes were considered to be abnormal if they met at least one of the following three criteria: (1) more than two staining spots present per cell; (2) the diameter of the centrosomes in the tumor was more than twice the diameter of those in nontumor cells; (3) the centrosomes were organized in patchy aggregates or elongated in stringlike structures greater than $3 \mu \mathrm{m}$ in length.

\section{DNA Ploidy Analysis}

The nuclear suspensions were obtained from each $60 \mu \mathrm{m}$ thick paraffin section by proteolytic digestion 
and homogenization, and were smeared on glass slides with an automatic smear maker (Auto Smear CF12C, Sakura Seiki, Tokyo, Japan). ${ }^{18}$ DNA was stained with DAPI for $1 \mathrm{~h}$ at room temperature. The nuclear fluorescence images of more than 500 cancer cells were preserved as digital images and presented as a histogram of DNA content, using IP Lab software.

The nuclear DNA content of normal lymphocytes on the same smears was determined in order to provide an internal standard of diploid DNA content (2C). If the main peak in the DNA histogram was within $2 \mathrm{C} \pm 0.2 \mathrm{C}$, the tumor was considered to be diploid, while the tumors exhibiting peaks outside this specific range were classified as nondiploid. The nondiploid tumors were further classified as either tetraploid if the peaks in more than $20 \%$ of the total number of cells were within $4 \mathrm{C} \pm 0.4 \mathrm{C}$, or aneuploid if the peaks were outside either of these specific ranges.

\section{Statistical Analysis}

Statistical significance was analyzed using the Fisher's exact probability test or the Mann-Whitney
$U$ nonparametric test. $P<0.05$ was considered statistically significant. Spearman's correlation coefficient was used to determine whether the percentage of cells with centrosome abnormality significantly correlated with the Ki-67-positive index.

\section{Results}

Centrosomes were detected in tissue sections by the use of a polyclonal antibody to pericentrin, which is a major component of the centrosome matrix. In normal liver tissue from the control patients, one centrosome per cell was detected as a round dot, usually in the vicinity of the nuclear membrane (Figure 1a). In the case of the HCC cells, several distinct patterns of centrosome abnormalities were detected, including supernumerary centrosomes (Figure 1b) and centrosomes with an abnormal shape and size (Figure 1c).

Centrosome aberrations were found in 30 out of 33 samples $(91 \%)$ from the tissues of HCC patients. The percentage of tumor cells with abnormal centrosomes was significantly higher in the nondiploid tumors $(15.8 \pm 15.9 \%$ ) than in the diploid tumors
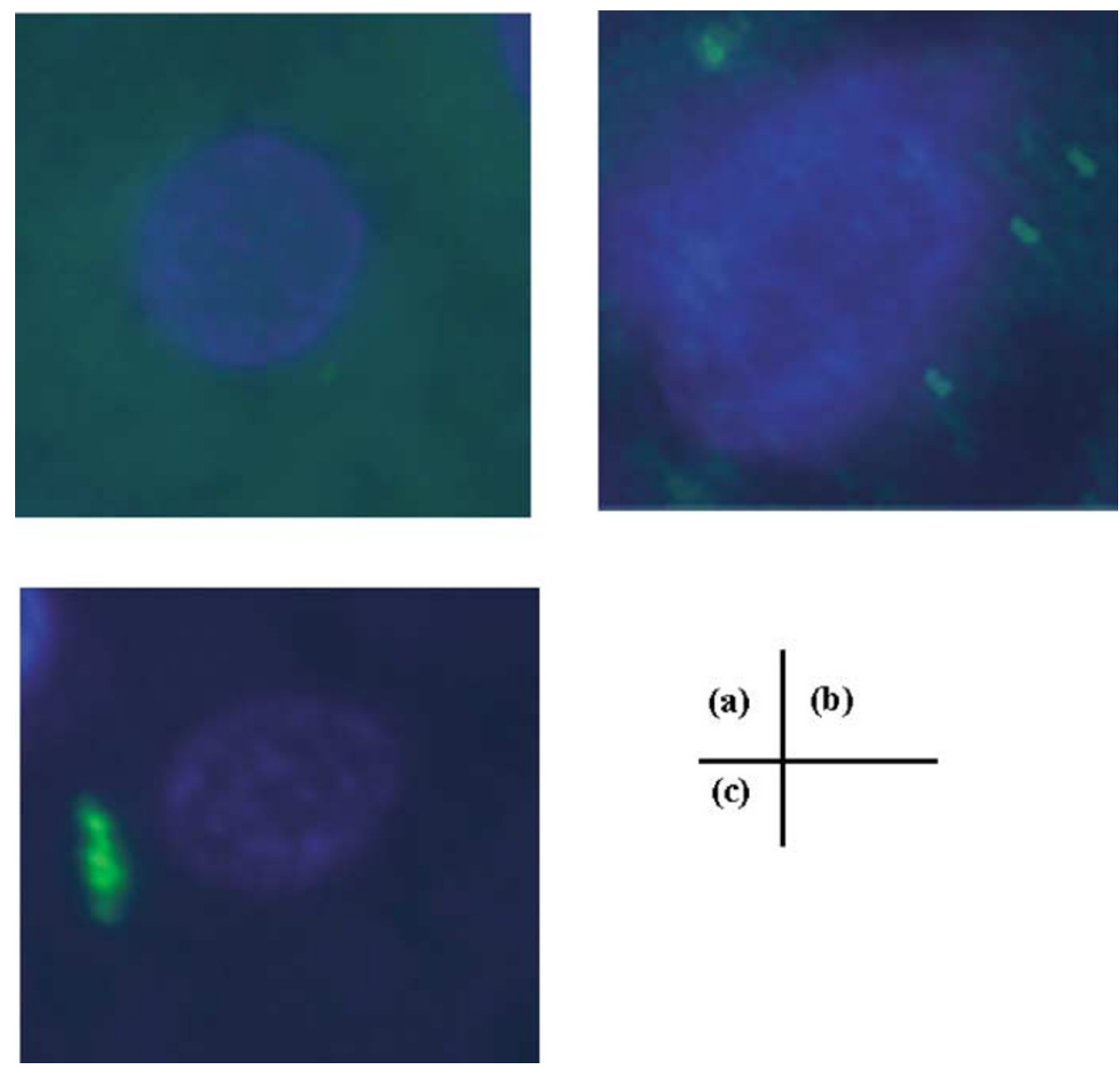

Figure 1 Different patterns of pericentrin immunostaining (green), counterstained with DAPI (blue) in liver samples. (a) Normal staining pattern (normal liver). One centrosome per cell can be identified as a dot, usually in the vicinity of the nuclear membrane. (b) Supernumerary centrosomes (HCC). (c) Centrosomes with abnormal shape and size (HCC). 
$(5.4 \pm 5.1 \%)(P<0.05$ by Mann-Whitney test, Figure 2 ), and tended to be higher in the tumors with p53 aberration $(11.6 \pm 13.1 \%$ o $)$ than in those with wildtype p53 (5.6 $\pm 6.8 \%$ ) (the $P$-value was less than 0.05 by Mann-Whitney test; when the single outlying value indicated by the arrowhead in Figure 3 was excluded, the $P$-value was 0.10 by one-tailed Student's t-test) (Figure 3). With respect to the clinical stages of HCC, there was no significant difference in the percentage of tumor cells with centrosome abnormalities between stage I-II and III-IV tumors. There were no significant differences in the percentage of tumor cells with centrosome abnormalities between any of two groups based on tumor size (A: $<3 \mathrm{~cm}$; $\mathrm{B}$ : $\geq 3 \mathrm{~cm}$ and $<5 \mathrm{~cm}$; $\mathrm{C}: \geq 5 \mathrm{~cm})$.

To establish if cancer cells with more than two centrosomes became dominant in a tumor following frequent cell division, we studied the relationship between the percentage of tumor cells with centrosome aberrations and the cell proliferative rate as measured by the Ki-67-positive index or tumor size. However, no significant correlations were detected between any of these factors.

With regard to our investigation into the possible correlation between p53 aberration and DNA ploidy, we found that nine out of the 11 non-diploid tumors exhibited p53 aberration (82\%) compared with only nine out of the 22 diploid tumors $(41 \%)$. This higher frequency of p53 aberration in non-diploid compared with diploid tumors was significant $(P<0.0001$ by Fisher's exact probability test, Figure 4).

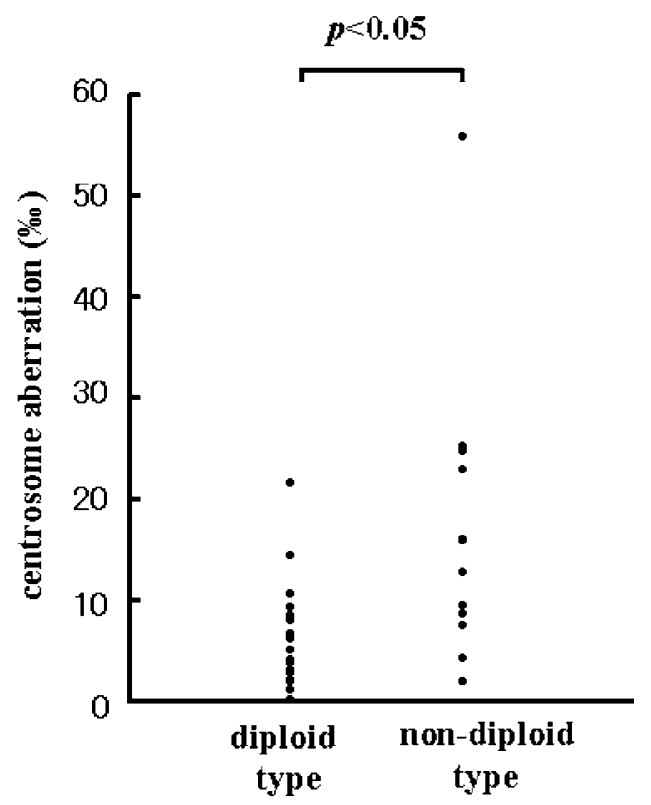

Figure 2 Comparison of the percentage of tumor cells with abnormal centrosomes in the nondiploid tumors and diploid tumors. A significant difference was detected. $(P<0.05$ by MannWhitney test).

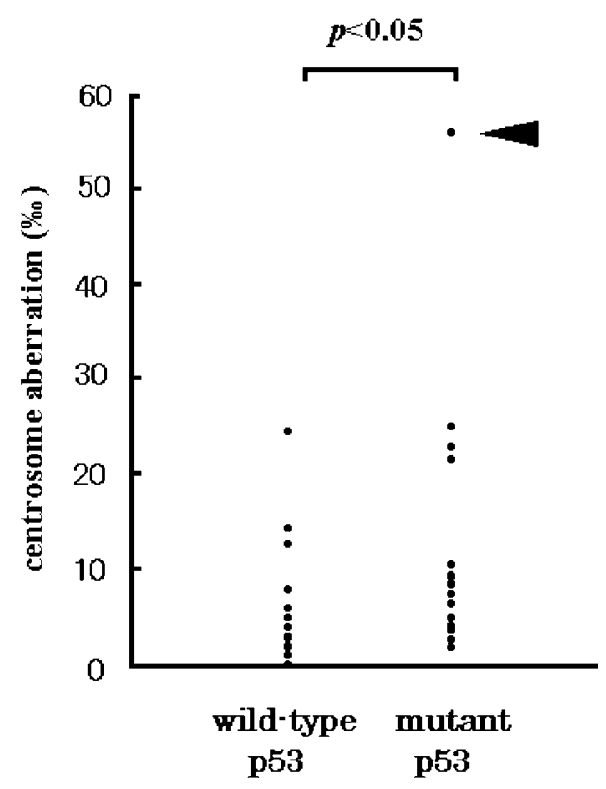

Figure 3 Comparison of the percentage of tumor cells with abnormal centrosomes in the tumors with p53 aberration and the tumors with wild-type p53. The $P$-value was less than 0.05 by the Mann-Whitney $U$ test. When the single outlying value indicated by the arrowhead was excluded, the $P$-value was 0.10 by onetailed Student's $t$-test.

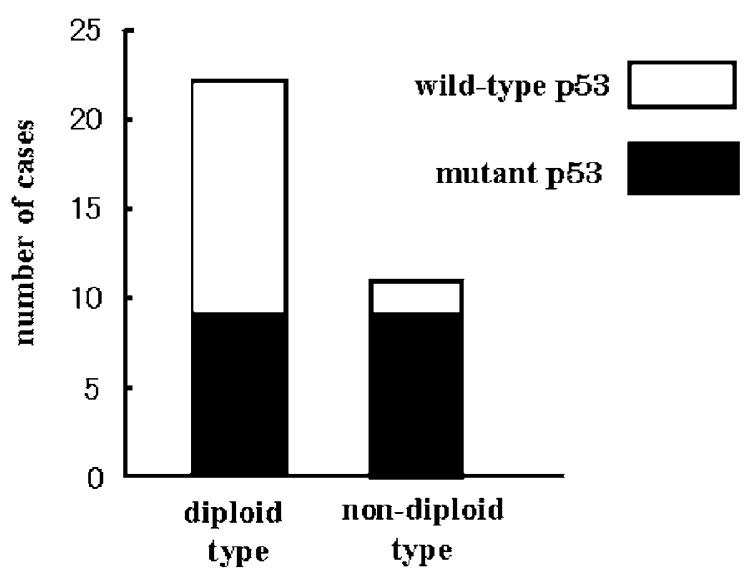

Figure 4 p53 aberration in diploid and nondiploid tumors. The frequency of p53 aberration among the nondiploid tumors was significantly higher than that among the diploid tumors $(P<0.0001$ by Fisher's exact probability test $)$.

\section{Discussion}

Normal liver tissue consists mostly of hepatocytes in the $G_{0}$, or resting, phase, that typically have only one centrosome per cell. Centrosome duplication, that facilitates the accurate division of chromosomes during mitosis, is initiated at the $\mathrm{G}_{1} / \mathrm{S}$ boundary and is completed during the $G_{2}$ phase, and under normal conditions will occur only once during each cell cycle. $^{1,2}$ This process of centrosome duplication is strictly regulated and can occasionally become disturbed in malignant cells. We have reported here, for the first time, the genetic instability of HCC in 
the clinical situation, with particular respect to centrosome aberration and p53 mutation.

Fukasawa et $a l^{19}$ first proposed the direct involvement of p53 inactivation in abnormal centrosome amplification. In this in vitro study, they showed that in mouse embryonic fibroblasts lacking the p53 protein, multiple copies of functionally competent centrosomes are duplicated during a single cell cycle. Furthermore, they showed that the centrosome duplication started much earlier than normal in the $G_{1}$ phase and that centrosome duplication was not controlled in a cell cycle-specific manner. In addition, it has been shown that spleen-, thymus-, and bone marrow-derived cells, as well as skin- and spleen-derived fibroblasts of p53-nullizygous (p53-/-) mice contained multiple centrosomes. ${ }^{10}$ Thus, it appears that the loss of cell cycle-specific regulation of centrosome duplication via the absence of p53 can, in part, account for the correlation between the occurrence of aberrant centrosomes and p53 mutation. Some investigators have suggested that centrosome aberration is tumor-related since in many different types of non-neoplastic cells that have varying degrees of proliferative activity, there were no centrosome abnormalities. ${ }^{4}$ This is supported by our result where we showed that the percentage of tumor cells with centrosome abnormalities was not correlated with the Ki-67-positive index.

In addition to direct involvement in centrosome amplification, p53 mutation also plays an indirect role. Besides mutant p53 protein, other factors are known to induce centrosome amplification such as errors in cell division which can lead to the development of hyperploid and multinucleated cells. ${ }^{11,20-22}$ Owing to the absence of the p53 suppressor protein, these hyperploid cells with extra centrosomes may survive and proliferate by escaping the $G_{1} / S$ and $G_{2} / M$ checkpoint systems, which normally would detect, repair or destroy cells exhibiting any chromosomal abnormality, such as ploidy. This results in the production of aneuploid progeny through unequal chromosomal segregation. $^{9,11}$ We have shown in our study that nondiploid tumors were generally associated with p53 aberration, and that could be correlated with centrosome aberration. This confirms previous work. ${ }^{23}$ Therefore, we speculate that liver cancer cells that have undergone centrosome amplification and p53 mutation have the potential to show genetic instability. However, because some nondiploid tumors did not show centrosome amplification and p53 mutation, it is possible that other factors are involved in genetic instability and over-riding cell cycle checkpoint systems.

In our study, genetic instability was evaluated by DNA image cytometry. We previously reported that the copy number of chromosome 17 is closely associated with DNA ploidy in HCC, as almost all $(87.5 \%)$ diploid tumors showed disomy of chromosome 17 whereas all of the nondiploid tumors showed trisomy or tetrasomy. ${ }^{18}$ Therefore, we speculate that about $10-15 \%$ of tumors diagnosed as diploid by DNA cytometry possibly harbored cells with increased copy number of chromosome 17. In addition, according to the report on the development of colorectal tumors, a few chromosomal changes were observed in some regions of diploid tumors before the emergence of nondiploidy. ${ }^{24}$ These reports and the results of our study suggest that in some diploid tumors, centrosome aberration and p53 mutation induce a small degree of chromosome instability that is not initially manifested by a change in the pattern of DNA ploidy.

According to previous reports, the incidence of abnormal centrosomes varies among different kinds of cancers. ${ }^{4,15}$ Our study showed that the incidence of abnormal pericentrin in HCC was not as high as that seen in other types of cancers. The presence of abnormal pericentrin can be used as a direct measure of centrosome abnormality. ${ }^{4,15}$ This is consistent with the low incidence of nuclear atypia among HCCs, especially among tumors that are well or moderately differentiated. In these differentiated types of HCC, there are subtle changes in nuclear and nucleolar features, and abnormal spindle patterns such as multiple bipolar spindles and asymmetric bipolar and multipolar spindles are seen only rarely. Similarly, centrosome abnormalities have been infrequently reported among endocrine tumors of the pancreas. ${ }^{25}$ and among prostate cancers,${ }^{15}$ both of which are characterized by a low incidence of nuclear atypia.

Although, the percentage of tumor cells with centrosome aberration in our cases was not as high as that seen in other cancers, nevertheless, centrosome aberration was found in almost all HCC tissues even in the early stage. In most cases, HCC tumors grow slowly in the early stages but sometimes the growth rate can accelerate in an unpredictable fashion at any stage. ${ }^{26}$ Acceleration of the growth rate might be related to centrosome aberration, irrespective of the size and stage of the tumor, resulting in the emergence of aneuploid progeny.

\section{References}

1 Wang RJ, Wissinger W, King EJ, et al. Studies on cell division in mammalian cells. VII. A temperaturesensitive cell line abnormal in centriole separation and chromosome movement. J Cell Biol 1983;96: 301-306.

2 Kramer A, Neben K, Ho AD. Centrosome replication, genomic instability and cancer. Leukemia 2002;16:767-775.

3 Kramer A, Ho AD. Centrosome aberrations and cancer. Onkologie 2001;24:538-544.

4 Pihan GA, Purohit A, Wallace J, et al. Centrosome defects and genetic instability in malignant tumors. Cancer Res 1998;58:3974-3985.

5 Pihan GA, Purohit A, Wallace J, et al. Centrosome defects can account for cellular and genetic changes 
that characterize prostate cancer progression. Cancer Res 2001;61:2212-2219.

6 Ciciarello M, Mangiacasale R, Casenghi M, et al. p53 displacement from centrosomes and p53-mediated G1 arrest following transient inhibition of the mitotic spindle. J Biol Chem 2001;276:19205-19213.

7 Tarapore P, Tokuyama Y, Horn HF, et al. Difference in the centrosome duplication regulatory activity among p53 'hot spot' mutants: potential role of Ser 315 phosphorylation-dependent centrosome binding of p53. Oncogene 2001;20:6851-6863.

8 Borel F, Lohez OD, Lacroix FB, et al. Multiple centrosomes arise from tetraploidy checkpoint failure and mitotic centrosome clusters in p53 and RB pocket protein-compromised cells. Proc Natl Acad Sci USA 2002;99:9819-9824.

$9 \mathrm{Xu} \mathrm{X}$, Weaver Z, Linke SP, et al. Centrosome amplification and a defective G2-M cell cycle checkpoint induce genetic instability in BRCA1 exon 11 isoform-deficient cells. Mol Cell 1999;3:389-395.

10 Fukasawa K, Wiener F, Vande Woude GF, et al. Genomic instability and apoptosis are frequent in p53 deficient young mice. Oncogene 1997;15: 1295-1302.

11 Meraldi P, Honda R, Nigg EA. Aurora-A overexpression reveals tetraploidization as a major route to centrosome amplification in p53-/- cells. EMBO J 2002; 21:483-492.

12 Lingle WL, Barrett SL, Negron VC, et al. Centrosome amplification drives chromosomal instability in breast tumor development. Proc Natl Acad Sci USA 2002; 99:1978-1983.

13 Verschuren EW, Klefstrom J, Evan GI, et al. The oncogenic potential of Kaposi's sarcoma-associated herpesvirus cyclin is exposed by p53 loss in vitro and in vivo. Cancer Cell 2002;2:229-241.

14 Murakami Y, Hayashi K, Hirohashi S, et al. Aberrations of the tumor suppressor p53 and retinoblastoma genes in human hepatocellular carcinomas. Cancer Res 1991;51:5520-5525.

15 Pihan GA, Wallace J, Zhou Y, et al. Centrosome abnormalities and chromosome instability occur together in pre-invasive carcinomas. Cancer Res 2003;63:1398-1404.
16 Kimura H, Kagawa K, Deguchi T, et al. Evaluation of proliferative activity in hepatocellular carcinoma by Ki-67 immunostaining of paraffin-embedded tissues after antigen retrieval. Int Hepatol Commun 1994;2: 99-104.

17 Kuo KK, Sato N, Mizumoto K, et al. Centrosome abnormalities in human carcinomas of the gallbladder and intrahepatic and extrahepatic bile ducts. Hepatology 2000;31:59-64.

18 Kimura H, Kagawa K, Deguchi T, et al. Cytogenetic analyses of hepatocellular carcinoma by in situ hybridization with a chromosome-specific DNA probe. Cancer 1996;77:271-277.

19 Fukasawa K, Choi T, Kuriyama R, et al. Abnormal centrosome amplification in the absence of p53. Science 1996;271:1744-1747.

20 Shekhar MP, Lyakhovich A, Visscher DW, et al. Rad6 overexpression induces multinucleation, centrosome amplification, abnormal mitosis, aneuploidy, and transformation. Cancer Res 2002;62:2115-2124.

21 Chen Z, Indjeian VB, McManus M, et al. CP110, a cell cycle-dependent CDK substrate, regulates centrosome duplication in human cells. Dev Cell 2002;3:339-350.

22 Goepfert TM, Brinkley BR. The centrosome-associated Aurora/Ipl-like kinase family. Curr Top Dev Biol 2000;49:331-342.

23 Qiao L, Wu P, Ghaleb $\mathrm{AH}$, et al. Bivariate flow cytometric analysis of p53 and DNA content in hepatocellular carcinoma. Anal Quant Cytol Histol 1994;16:124-130.

24 Katsura K, Sugihara H, Nakai S, et al. Alteration of numerical chromosomal aberrations during progression of colorectal tumors revealed by a combined fluorescence in situ hybridization and DNA ploidy analysis of intratumoral heterogeneity. Cancer Genet Cytogenet 1996;90:146-153.

25 Sato N, Mizumoto K, Nakamura M, et al. Centrosome abnormalities in pancreatic ductal carcinoma. Clin Cancer Res 1999;5:963-970.

26 Nakajima T, Moriguchi M, Mitsumoto Y, et al. Simple tumor profile chart based on cell kinetic parameters and histologic grade is useful for estimating the natural growth rate of hepatocellular carcinoma. Hum Pathol 2002;33:92-99. 